\title{
A COMPOSITE CATAlogue OF ASTEROID PROPER ELEMENTS
}

\author{
ANDREA MILANI \\ Dipartimento di Matematica, Via Buonarroti 2, I-56127 Pisa, Italia \\ E-mail milani@dm.unipi.it \\ EDWARD BOWELL \\ Lowell Observatory, 1400 W. Mars Hill Road, Flagstaff AZ 86001, USA \\ $E$-mail elgb@lowell.edu \\ ZORAN KNEŽEVIĆ \\ Astronomska opservatorija, Volgina 7, 11050 Beograd, Yugoslavia \\ ANNE LEMAITRE \\ Dép. Mathématique, FUNDP, Rempart de la Vierge 8, B-5000 Namur, Belgium \\ $E$-mail alemaitr@math.fundp.ac.be \\ ALESSANDRO MORBIDELLI \\ Observatoire de la Côte d'Azur, BP 229, 06304 Nice Cedex 04, France \\ $E$-mail morby@rameau.obs-nice.fr \\ KARRI MUINONEN \\ Observatorio, PL 14, 00014 Helsingin yliopisto, Suomi-Finland \\ E-mail karri@ceres.helsinki.fi
}

\section{Contents of the catalogue and sources}

We have assembled the asteroid proper elements computed by different authors, using different methods, and for different regions of the asteroid belt. Asteroids on planet crossing orbits are not yet included. The use of very different algorithms is dictated by the dynamics of the different regions. For the asteroids of the main belt, having semimajor axes between 2.1 and $3.8 \mathrm{AU}$, and with proper eccentricity and sine of inclination less than 0.3 , proper elements are computed by a fully analytical iterative theory developed by Milani and Knežević $(1990,1992,1994$; hereafter $\mathrm{M} \& \mathrm{~K})$. For high inclination and/or eccentricity main-belt asteroids, having $1.8<a<3.8 A U$ and either $e$ or $\sin I$ larger than 0.24 , proper elements are computed by a semianalytical theory developed by Lemaitre and Morbidelli (1994; hereafter L\&M). For Trojans, proper elements are computed by a synthetic theory (that is, from the output of a numerical integration for a few $M y r$ ) by Milani (1993). For the Hilda asteroids in the $3: 2$ resonance, proper elements have been computed by a synthetic theory by Schubart $(1982,1991)$. For a discussion of the state of the art in the computation of proper elements, see Knežević and Milani, this volume; for Trojans, see Milani, this volume; for some other cases (e.g. proper 
elements for resonant asteroids), see Froeschlé and Morbidelli, this volume. When and if new proper elements will be available for other asteroids, they will be added to the public domain file, together with updates and upgrades of the existing catalogues.

Users of this composite catalogue need to be aware that different authors use different definitions of the proper elements. For the main belt, mean semimajor axes are computed by analytical removal of short periodic perturbations with an algorithm developed by Knežević (1988), Milani and Knežević $(1990,1994)$ (the output of this intermediate computation is a catalogue of mean elements, also available); this mean semimajor axis is used as proper $a$ by both M\&K and L\&M. For Trojans and Hildas, the mean semimajor axis is not useful; the meaningful proper element is the amplitude of the oscillation of the semimajor axis around the mean value; as an alternative, the amplitude of the libration of the critical argument can be used. For proper eccentricities and sine of inclination, the values computed by $M \& K$ can be interpreted as the average values over a time much longer than the time scale of the secular perturbations; in contrast, L\&M give two values for both proper $e$ and $\sin I$, which can be interpreted as maximum and minimum value (over one cycle of the argument of perihelion). The arithmetic mean of the two values given by $L \& M$ is close, at least for moderate inclinations, to the $M \& K$ value. For Trojans and Hildas, proper $e$ and $\sin I$ have a geometric interpretation similar to that of the $M \& K$ elements, although the computational procedure is completely different.

In most cases, the use of proper elements defined in different ways (e.g. to identify asteroid families) should not give rise to problems, because different definitions are used in widely separated regions. However, the $M \& K$ and the L\&M elements do have an overlap region (for eccentricity and/or sine of inclination between 0.24 and 0.3). In this region the two methods have comparable accuracy; the overlap is necessary for asteroid family studies, to avoid loss of information on families extending across this range; the users are warned that to take a difference between $M \& K$ and $L \& M$ asteroids might result in a significant loss of accuracy (with respect to differences between elements computed by means of the same algorithm).

\section{Accuracy and reliability}

Proper elements have been computed for essentially all the asteroids for which the available osculating elements are accurate enough (this does not apply to the Hildas, for which the catalogue is not entirely up to date). We have included all the numbered asteroids, together with the asteroids observed at more than one opposition. For the orbits computed on the basis of observations performed at one opposition only, we have used the covariance matrices of the orbital elements computed by Muinonen and Bowell (1993; see also Muinonen et al., 1994) to assess their precision. If we assume that the main purpose of the proper elements is to study asteroid families, then for each metric used to compute the distance between two asteroids in the proper elements space there is a corresponding covariance, which can be computed as a function of the full covariance matrix of the elements. If the metric is the simplest possible one (for a discussion of the metric problem, see Zappalà et al. 1990, Milani et al. 1992) : 


$$
d=\sqrt{\frac{1}{4}\left(\frac{\delta d}{a}\right)^{2}+2(\delta e)^{2}+2(\delta \sin I)^{2}}
$$

then the covariance to be used is :

$$
\iota_{0}^{2}=e^{2}\left(\Sigma_{\omega \omega}+\Sigma_{\Omega \Omega}+2 \Sigma_{\omega \Omega}\right)+\Sigma_{e e}+\Sigma_{\Omega \Omega} \sin ^{2} i+\Sigma_{i i} \cos ^{2} i+\Sigma_{a a} \frac{1}{4 a^{2}} .
$$

where the $\Sigma$.. are the Bowell and Muinonen covariances. We have therefore computed proper elements for the single opposition orbits with $\iota_{0} \leq 0.01$; orbits determined in a poorer way (e.g., by observations over a very short arc) would not be useful to find families, since the error in the proper elements cannot be smaller than the error in the osculating ones. For single-opposition orbits used in this catalogue, the quality of the osculating elements is indicated by a Quality Code for Osculating elements (QCO), on a decibel scale in which $Q C O=0$ corresponding to $\iota_{0}=10^{-4}$ and $Q C O=20$ corresponding to $\iota_{0}=10^{-2}$.

As a result, the current version of the osculating elements file used as input catalogue for the computation of mean elements contains 14127 orbits. Mean elements have been computed for 13456 main belt asteroids. The proper elements M\&K catalogue contains 12573 asteroids; L\&M contains 2511 asteroids, the Trojans are 184 and the Hildas 57 . The proper elements files currently amount to about $1150 \mathrm{~KB}$; the mean elements, the input catalogue of osculating elements, the software and the auxiliary files amount to about $4 M B$.

The accuracy and reliability of the computation of the mean and proper elements are indicated by other quality codes. A Quality Code for Mean elements (QCM) indicates the cases in which the removal of short periodic perturbations results in degraded accuracy because of some nearby low-order mean-motion resonance. For the M\&K proper elements, a Resonance FLag (RFL) indicates the cases in which the results might be degraded by some secular resonance; the RFL encodes the small divisor responsible for the divergence of the iterative theory. For the $L \& M$ proper elements, the flag $I C=0$ means that the process is convergent, $I C \neq 0$ gives by its value an indication about the non convergence reason (see the documentation for more details); the flag $I L=0$ indicates a perihelion circulation case and $I L= \pm 1$ a libration case. For the Trojans, a separate file contains estimates of the stability for all the proper elements and frequencies, as obtained by the running box method.

\section{Public-domain software}

Together with the data files, the distribution files contain also the software used to produce some of the data. In particular, for the $M \& K$ mean and proper elements, all the software is already public domain and the users should be able to compute mean and proper elements by themselves. Although transportability cannot always be guaranteed, the computer programs are written in standard FORTRAN 77; they are reasonably robust and have been tested on several different CPUs. For the UNIX environment, a makefile is provided, and compilation should be straightforward. Some macros for compilation in a DOS environment are available upon request. 
To test the stability of the proper elements, at least for low-eccentricity orbits, the numerical integration software package orbit8 $\mathrm{v}$ is also provided. Some essential documentation is available, both as README files and as $\mathrm{T}_{\mathrm{EX}} \mathrm{X}$ sources; however, the users should remember that this is not commercial software, and documentation might not be always complete.

\section{Access}

The catalogues of proper elements, the auxiliary files and the public domain software are available by anonymous $f t p$ to the server of the Department of Mathematics, University of Pisa, at the internet address gauss.dm.unipi.it (absolute address 131.114.6.55). The subdirectory is pub/propel.

The complete distribution file (with a name like versNNN.tar.Z) has been packed and compressed (with the standard UNIX utilities tar and compress); the size is currently about $3 M B$. The users of different operating systems should get the uncompressed files they need one by one, by looking in the subdirectories propels, meanels, oscels, sources, doc. In case of any problem, please contact the relevant author by $\mathrm{E}$-mail.

\section{References}

Knežević, Z. 1988. "Asteroid mean orbital elements". Bull. Astron. Obs. Belgrade 139, 1-6.

Lemaitre, A., and Morbidelli, A. 1994. "Calculation of proper elements for high inclined asteroidal orbits". Celest. Mech., in press.

Milani, A. 1993. "The Trojan asteroid belt : proper elements, stability, chaos and families". Celest. Mech., 57, 59-94.

Milani, A., and Knežević, Z. 1990. "Secular perturbation theory and computation of asteroid proper elements". Celestial Mechanics 49, 347-411.

Milani, A., and Knežević, Z. 1992. "Asteroid proper elements and secular resonances". Icarus, 08, 211-232.

Milani, A., and Knežević, Z. 1994. "Asteroid proper elements and the dynamical structure of the asteroid main belt". Icarus, in press.

Milani, A., Farinella, P., and Knežević, Z. 1992. "On the search for asteroid families". In Interrelations between physics and dynamics for minor bodies in the solar system (D. Benest, C. Froeschléi, Eds.), 85-132, Editions Frontières, Gif-sur-Yvette.

Muinonen, K., and Bowell, E. 1993. "Asteroid orbit determination using Bayesian probabilities". Icarus 104, 255-279.

Muinonen, K., Bowell, E. and Wasserman, L.H. 1994. "Orbital uncertainities of singleopposition asteroids." Planet. Space Sci., in press.

Schubart, J. 1982. "Three characteristic parameters of orbits of Hilda type asteroids". Astron. Astrophys. 114, 200-204.

Schubart, J. 1991. "Additional results on orbits of Hilda type asteroids". Astron. Astrophys. 241, 297-302.

Zappalà, V., Cellino, A., Farinella, P., and Knežević, Z. 1990. "Asteroid families I : identification by hierarchical clustering and reliability assessment". Astron. J. 100, 20302046. 